\title{
MiR-378a-5p inhibits angiogenesis of oral squamous cell carcinoma by targeting KLK4
}

\author{
Z. CUI ${ }^{1, *}$, Q. L. LIU', S. Q. SUN², K. JIAO ${ }^{1}$, D. R. LIU' ${ }^{1}$, X. C. ZHOU ${ }^{3}$, L. HUANG ${ }^{3}$ \\ ${ }^{1}$ Department of Oral and Maxillofacial Surgery, School and Hospital of Stomatology, Jilin University, Changchun 130021, China; ${ }^{2}$ Department \\ of Prosthodontics, School and Hospital of Stomatology, Jilin University, Changchun 130021, China; ${ }^{3}$ Department of Orthodontics, School and \\ Hospital of Stomatology, Jilin University, Changchun 130021, China
}

*Correspondence: wilmei@163.com

Received March 6, 2019 / Accepted June 19, 2019

\begin{abstract}
Oral squamous cell carcinoma (OSCC) is still a leading cause of cancer death owing to distant metastasis, which is largely facilitated by tumor angiogenesis. MicroRNA (miR)-378a-5p and Kallikrein-related peptidase 4 (KLK4) participate in tumorigenesis and tumor metastasis according to previous studies, yet the exact role they play in tumor angiogenesis remains poorly understood. The aim of the present study was to investigate the effect of miR-378a-5p and KLK4 on angiogenesis of OSCC. MTT assay showed that the expression level of miR-378a-5p was negatively correlated with the proliferation of OSCC cells. ELISA and western blot assay showed that downregulation of miR-378a-5p promoted VEGF expression. Tube formation and in vivo chicken chorioallantoic membrane (CAM) assay showed that inhibition of miR-378a-5p reduced tube formation of human umbilical vein endothelial cells (HUVECs) and newly formed microvessel. On the contrary, overexpression of KLK4 enhanced angiogenesis of OSCC cells with increased VEGF expression, tube formation activity of HUVECs and newly formed microvessel. Moreover, the dual-luciferase assay validated that KLK4 was a target gene of miR-378a-5p. MiR-378a-5p silencing induced tube formation was suppressed by the downregulation of KLK4. Besides, the activation of Wnt/ $\beta$-catenin signaling pathway in miR-378a-5p antagomir transfected cells was also blocked by the KLK4 shRNA. To sum up, our study suggests that miR-378a-5p suppressed angiogenesis of OSCC at least partly by the regulation of KLK4.
\end{abstract}

Key words: miR-378a-5p, KLK4, oral squamous cell carcinoma, angiogenesis, Wnt/ $\beta$-catenin signaling

Oral carcinoma, an epithelial carcinoma occurring at oral mucosa, is one of the most common malignant tumors among head and neck tumors, which has serious impacts on the daily life of human beings [1]. Oral squamous cell carcinoma (OSCC) accounts for more than $90 \%$ of all oral tumors [2], despite the great strides made in surgery, radiotherapy and chemotherapy, the overall five-year survival rate of patients with advanced OSCC is only about $50 \%$ owing to distant metastasis [3]. Tumor angiogenesis plays a crucial role in tumorigenesis and tumor development, it provides oxygen and other essential nutrients for tumor microenvironment and therefore facilitates tumor distal metastasis. Thus a better understanding of tumor angiogenesis is of great importance for cancer therapy $[4,5]$.

MicroRNAs are endogenous small non-coding RNAs with an average of 22 nucleotides in length. MicroRNAs are involved in numerous physiological processes such as cell differentiation, cell proliferation and cancer devel- opment. MicroRNA-378a (miR-378a) locates in the first intron of the ppargclb gene, miR-378a-5p is the passenger strand derived from the pre-miR378a [6]. The sequence of miR-378a is highly conserved between species as the miR-378a-5p strand is completely identical in human and mice. In recent studies, miR-378a was reported to participate in metabolism, muscle cell development and tumor angiogenesis, and miR-378a was underexpressed in OSCC $[7,8]$

Human kallikrein-related peptidases (KLKs), a subgroup of serine proteases, are highly conserved in gene structures and protein sequences. Increasing evidence shows that KLKs are implicated in a variety of physiological processes including carcinogenesis, tumor metastasis and angiogenesis [9, 10]. For instance, kallikrein gene transfer could enhance the expression of vascular endothelial growth factor (VEGF) to induce the angiogenesis in the early period after cerebral ischemia/reperfusion [11]. KLK5 
can digest plasminogen and generate angiostatin 4.5, and further inhibit angiogenesis [12].

KLK4, first purified from pig teeth in Japan, was expressed during the maturation stage of enamel development, its protease activity was later found to be inhibited by phenylmethylsulfonyl fluoride (PMSF) [13]. In recent studies, KLK4 expression was reported to be related to poor prognosis of prostate and ovarian cancers [14]. Besides, KLK4 could promote cancer-associated fibroblasts (CAF) differentiation, which is crucial for cancer progression. KLK4 has higher expression in premalignant and malignant prostatic lesions compared to normal prostate epithelia. KLK4 could also increase the expression of VEGF and stimulate human umbilical vein endothelial cells (HUVECs) proliferation [15]. These results indicate that KLK4 might get involved in tumor angiogenesis. KLK4 is a candidate target of miR-378a predicted by bioinformatics analysis, thus we investigated the correlation between KLK4 and miR-378a, and their impact on tumor angiogenesis of OSCC.

In this study, we investigated the effect of miR-378a and KLK4 on the tumor angiogenesis of OSCC, validated the target binding of miR-378a to 3'-UTR of KLK4, and analyzed the role of $\mathrm{Wnt} / \beta$-catenin signaling pathway in miR-378amediated tumor angiogenesis.

\section{Materials and methods}

Cells and reagents. Human umbilical vein endothelial cells (HUVECs) were purchased from Shanghai Zhong Qiao Xin Zhou Biotechnology Co. (China), Tca-8113 cells were purchased from the cell bank of Chinese Academy of Sciences (China), TSCCA and SCC-25 cells were purchased from Procell (China). Primary antibodies used in western blot were as follows: KLK4 (1:200), VEGFA (1:1000) and Wnt1 (1:500) antibodies (Abcam, UK), p- $\beta$-catenin (1:1000) and $\beta$-catenin (1:1000) antibodies (CST, USA), KLK4 (1:100, Bioss, China) antibody used in immunofluorescence assay. Histone H3 (1:5000, Gene Tex, USA) and GAPDH (1:10000, Proteintech, China) were used as internal control. Cy3-labeled goat anti-rabbit IgG (Beyotime, China), HRP-conjugated goat anti-rabbit IgG and HRP-conjugated goat anti-mouse IgG (1:3000, Solarbio, China) were used as secondary antibodies. Cell culture medium was purchased from Gibco (USA). Luciferase assay kit was purchased from KeyGEN (China). MiR-378a-5p (ID: MIMAT0000731) agomir and antagomir were purchased from Gene Pharma (China). The coding sequence of KLK4 was inserted into pcDNA3.1 (Invitrogen, USA) between BamH I and Xho I to overexpress KLK4. KLK4 and NC short hairpin RNA fragments were inserted into pRNA-H1.1 (Genescript, USA) between BamH I and Hind III. Sequences of shRNAs used in this study were as follows:

KLK4 shRNA sense (5'-3'): GATCCCCGCACACTGTTTCCAGAACTTTCAAGAGAAGTTCTGGAAACAGTGTGCTTTTT; KLK4 shRNA antisense $\left(5^{\prime}-3^{\prime}\right)$ :
AGCTAAAAAGCACACTGTTTCCAGAACTTCTCTTGAAAGTTCTGGAAACAGTGTGCGGG; NC shRNA sense (5'-3'): GATCCCCTTCTCCGAACGTGTCACGTTTCAAGAGAACGTGACACGTTCGGAGAATTTTT; NC shRNA antisense (5'-3'): AGCTAAAAATTCTCCGAACGTGTCACGTTCTCTTGAAACGTGACACGTTCGGAGAAGG.

Cell culture and transfection. TCA-8113 and TSCCA cells were cultured in Roswell Park Memorial Institute (RPMI)-1640 culture medium with 10\% fetal bovine serum (FBS), SCC-25 cells were cultured in Dulbecco's modified Eagle medium (DMEM)/F12 with 10\% FBS, HUVECs were cultured in DMEM with $10 \%$ FBS, all cells were cultured in a humidified chamber with $5 \% \mathrm{CO}_{2}$ at $37^{\circ} \mathrm{C}$. Cells were transfected with shRNAs or plasmids using Lipofectamine 2000 (Invitrogen, USA) following the manufacturer's instruction.

MTT assay. Cells were seeded in into 96-well plate $\left(4 \times 10^{3} \mathrm{cells} /\right.$ well $)$ and cultured for $24 \mathrm{~h}$. Cells were transfected and analyzed with MTT at $0 \mathrm{~h}, 12 \mathrm{~h}, 24 \mathrm{~h}, 48 \mathrm{~h}, 72 \mathrm{~h}$ and $96 \mathrm{~h}$ after transfection. Cells were incubated with $0.5 \mathrm{mg} / \mathrm{ml}$ MTT (KeyGEN, China) at $37^{\circ} \mathrm{C}$ for $4 \mathrm{~h}$, then were lysed with 150 $\mu \mathrm{l}$ DMSO for $10 \mathrm{~min}$ and the optical density value at $570 \mathrm{~nm}$ was measured with a microplate reader.

Quantitative real-time PCR. Total RNAs of cells were extracted and reverse-transcribed into cDNA using Super M-MLV reverse transcriptase (Bioteke, China). In particular, stem-loop RT primers were used for miR-378a-5p reversetranscription. Real-time PCR was performed using SYBR Green (Solarbio, China) in a fluorescent quantitative PCR Machine (BIONEER, Korea) following the manufacturer's instruction, and data were analyzed using the $2^{-\triangle \Delta C T}$ method. Stem-loop RT primers and real-time PCR primers used in this study were as follows:

hsa-miR-378a-5p specific stem-loop primer: 5'-GTTGGCTCTGGTGCAGGGTCCGAGGTATTCGCACCAGAGCCAACACACAG-3'; Has-U6-F specific stem-loop primer: 5'-GTTGGCTCTGGTGCAGGGTCCGAGGTATTCGCACCAGAGCCAACAAAATATGG-3'; hsa-miR-378a5p-F: 5'-CCGCTCCTGACTCCAGGTC-3'; hsa-miR-378a5p-R: 5'-GTGCAGGGTCCGAGGTATTC-3'; Has-U6-F: 5'-GCTTCGGCAGCACATATACT-3'; Has-U6-R: 5'-GTGCAGGGTCCGAGGTATTC-3'; KLK4-F: 5'ATCCGCAGTGGGTGCTGTCA-3';KLK4-R:5'-TGGCTCCCTGGCTCTTGGTC-3'; GAPDH-F: 5'-GAAGGTCGGAGTCAACGGAT-3'; GAPDH-R: 5'-CCTGGAAGATGGTGATGGGAT-3'.

Western blot. Proteins were extracted and quantified using the BCA protein assay kit (Beyotime, China). SDS-PAGE was carried out to fractionate proteins. Proteins were transferred to PVDF membranes (Millipore, USA) and blocked with 5\% skim milk. Then the PVDF membranes were incubated with primary antibodies overnight at $4^{\circ} \mathrm{C}$ and secondary antibodies for $1 \mathrm{~h}$ at room temperature.

Tube formation assay. Matrigel was thawed at $4{ }^{\circ} \mathrm{C}$ overnight and coated in 96-well plate without a bubble. HUVECs $\left(1.5 \times 10^{4}\right.$ cells/well $)$ were seeded into a coated 
96-well plate and cultured with conditioned culture medium for $48 \mathrm{~h}$ at $37^{\circ} \mathrm{C}$. Typical images were taken under a microscope ( $\times 100$ magnification).

Chicken chorioallantoic membrane assay. Breeding eggs of 7-10 days old were sterilized with $75 \%$ alcohol and incubated in a thermostat at $37 \pm 0.5^{\circ} \mathrm{C}$. A small hole was drilled on the surface of the air chamber and eggshell surrounding the hole was gently removed. The inner shell membrane was slightly torn off to expose the chorioallantoic membrane. The gelatin sponge (about $5 \mathrm{~mm} \times 5 \mathrm{~mm} \times 5 \mathrm{~mm}$ ) was selected as the carrier and placed on the avascular allantoic membrane. Conditioned culture medium was dripped on the carrier and photographs were taken 3 after days.

Dual-luciferase assay. Reporter plasmids carrying KLK4 3'-UTR or KLK4 3'-UTR mutant were co-transfected with NC agomir or miR-378a-5p agomir into 293T cells, the binding activity of miR-378a-5p to KLK4 was evaluated by measuring the normalized luciferase activity (firefly luciferase activity/Renilla luciferase activity).

Immunofluorescence assay. Cell slides were fixed with $4 \%$ paraformaldehyde for $15 \mathrm{~min}$, and $0.1 \%$ Triton X-100 was added to break cell membrane. Cell slides were incubated with goat serum (Solarbio, China) at room temperature for $15 \mathrm{~min}$, and then incubated with KLK4 antibody (1:100, Bioss, China) at $4{ }^{\circ} \mathrm{C}$ overnight. Afterward, cell slides were washed and incubated with Cy3-labeled secondary antibody for $60 \mathrm{~min}$ at room temperature, cells slides were then stained with DAPI and sealed with anti-fluorescent quenching reagent. Photographs were taken under a microscope $(\times 400$ magnification).

Statistical analysis. All data analysis was carried out using GraphPad Prism 7 (USA). All results were given as means \pm $\mathrm{SD}$ and mean values were compared by Student's t-test and One-way ANOVA. A p-value $<0.05$ was regarded as statistically significant.

\section{Results}

MiR-378a-5p inhibits tumor angiogenesis of OSCC. Expression of miR-378a-5p in Tca-8113 cells was obviously higher than that in TSCCA and SCC-25 cells (Figure 1A). The expression of miR-378a-5p in Tca-8113 cells was downregulated with miR-378a-5p antagomir transfection (Figure $1 \mathrm{~B}, \mathrm{p}<0.01$ ). The proliferation of Tca- 8113 cells was remarkably enhanced $24 \mathrm{~h}$ after miR-378a-5p antagomir transfection in contrast with control (Figure $1 \mathrm{C}, \mathrm{p}<0.01$ ). The content of VEGF and protein level of VEGFA in Tca-8113 cells were significantly increased after miR-378a-5p antagomir transfection (Figure 2A, 2B, $\mathrm{p}<0.01$ ). Tube formation of HUVECs was enhanced by conditioned medium of Tca-8113 cells containing miR-378a-5p antagomir (Figure $2 \mathrm{C}, \mathrm{p}<0.01$ ). The number of newly formed microvessels was remarkably increased by conditioned medium of Tca-8113 cells containing miR-378a-5p antagomir by CAM assay (Figure 2D). Besides, we upregulated the miR-378-5p with the specific agomir in TSCCA cells (Figure S1A), and the MTT assay showed that cell proliferation was suppressed by the upregulation of miR-378-5p (Figure S1B). The conditioned medium from miR-378-5p agomir transfected TSCCA cells significantly reduced tube formation activity of HUVECs compared to that of NC agomir transfected cells (Figure S1C).

KLK4 enhances tumor angiogenesis of OSCC. The mRNA and protein levels of KLK4 in Tca-8113 cells is significantly lower than that in TSCCA and SCC-25 cells (Figure $3 \mathrm{~A}, \mathrm{p}<0.01$ ). The mRNA and protein levels of KLK4 in Tca-8113 cells were conspicuous upregulated with KLK4 overexpression plasmid transfection (Figure 3B, 3C, p <0.01). The content of VEGF and protein level of VEGFA in Tca-8113 cells were significantly increased with KLK4 overexpression plasmid transfection (Figure $4 \mathrm{~A}, 4 \mathrm{~B}, \mathrm{p}<0.01$ ). Tube formation of HUVECs was enhanced by conditioned medium of Tca-8113 cells transfected with KLK4 overexpression plasmid (Figure 4C, $\mathrm{p}<0.01$ ). By CAM assay, the amount of newly formed microvessel was obviously increased by conditioned medium of Tca-8113 cells transfected with KLK4 overexpression plasmid (Figure 4D). The expression of KLK4 in TSCCA cells was further inhibited by a specific shRNA (Figure S1D, S1E), and the conditioned medium from KLK4
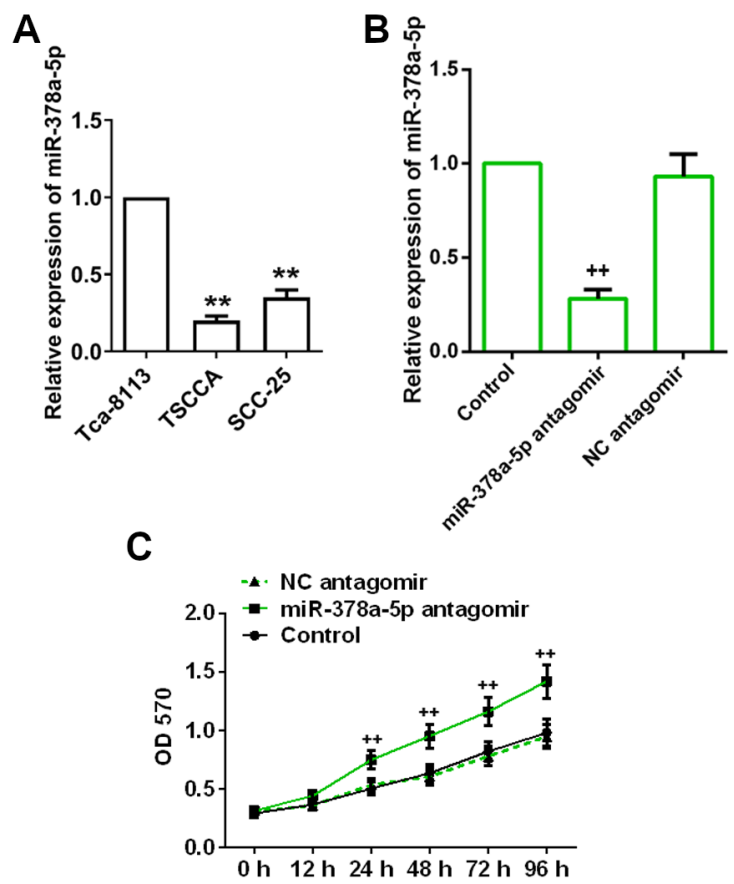

Figure 1. Expression of miR-378a-5p in OSCC cells and its effect on cell proliferation. A) The expression of miR-378a-5p in Tca-8113, TSCCA and SCC- 25 cell lines were evaluated by quantitative real-time PCR. B) The expression of miR-378a-5p antagomir or NC antagomir transfected Tca-8113 cells were evaluated by quantitative real-time PCR. C) MTT assay was used to detect cell proliferation of Tca-8113 cells. All data were presented as mean \pm SD. ${ }^{\star *} \mathrm{p}<0.01$ vs. Tca-8113 cells; ${ }^{++} \mathrm{p}<0.01$ vs. control cells. 
A

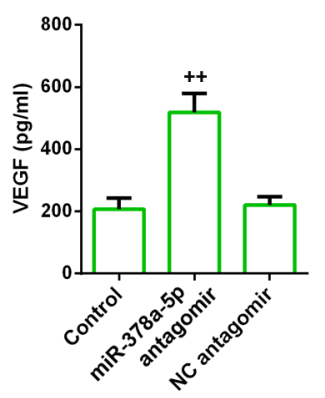

B

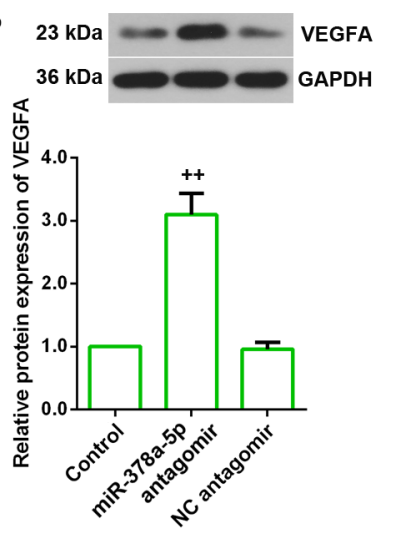

C

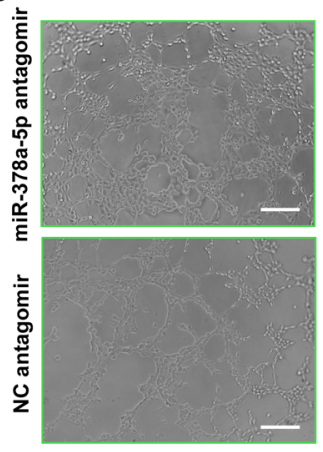

D

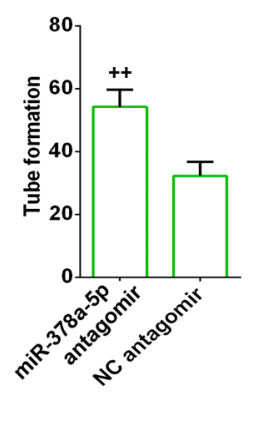

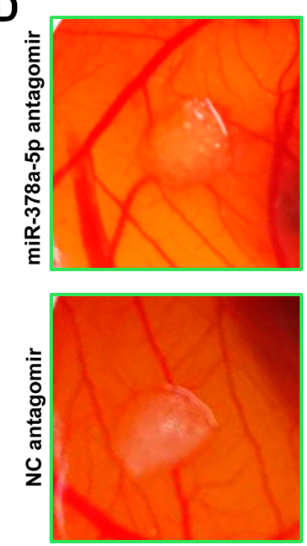

Figure 2. Effect of miR-378a-5p on angiogenesis. A) Contents of VEGF in Tca-8113 cells were measured by the commercial kit. B) The protein level of VEGFA in Tca-8113 cells were determined by western blot assay. C) HUVEC were treated with the conditioned medium from miR-378a-5p antagomir or NC antagomir transfected Tca-8113 cells, and the angiogenesis activity was detected by tube formation assay. D) The chicken chorioallantoic membrane $(\mathrm{CAM})$ assay was used to evaluate angiogenesis. All data were presented as mean $\pm \mathrm{SD} .{ }^{++} \mathrm{p}<0.01 \mathrm{vs}$. control cells.

A
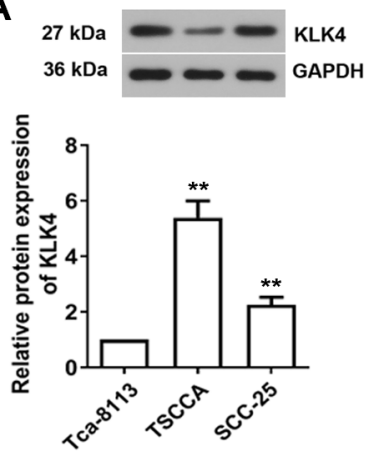

B

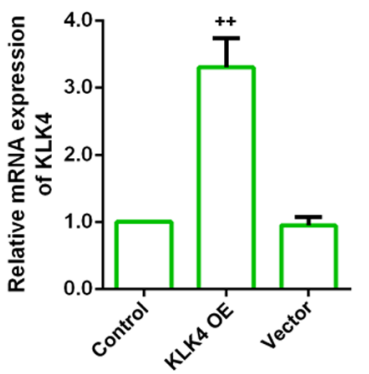

$27 \mathrm{kDa}$
$36 \mathrm{kDa}$

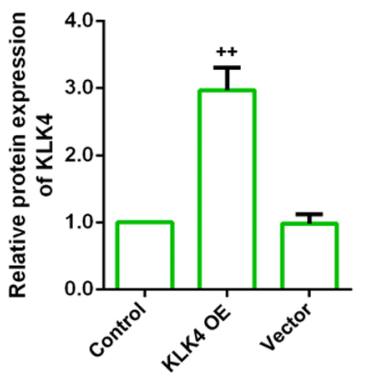

Figure 3. Expression of KLK4 in OSCC cells lines. A) The protein level of KLK4 in Tca-8113, TSCCA and SCC-25 cells were evaluated by quantitative real-time PCR. B) The mRNA level of KLK4 in over-expression vectors transfected Tca- 8113 cells was evaluated by quantitative real-time PCR. C) The protein level of KLK4 in over-expression vectors transfected Tca-8113 cells was determined by western blot assay. All data were presented as mean \pm SD. ${ }^{* *} p<0.01$ vs. Tca- 8113 cells.; ${ }^{++} p<0.01$ vs. control cells.

shRNA transfected TSCCA cells significantly reduced tube formation activity of HUVECs compared to that of NC shRNA transfected cells (Figure S1F).
KLK4 is a target gene of miR-378a-5p. The mRNA and protein levels of KLK4 in miR-378a-5p antagomir transfected Tca-8113 cells were significantly elevated compared to that of control cells (Figure 5A, p <0.01). On the contrary, the mRNA and protein levels of KLK4 were strongly reduced in miR-378a-5p agomir transfected TSCCA cells (Figure S2A, S2B). By immunofluorescence assay, miR-378a-5p antagomir transfected Tca-8113 cells showed higher KLK4 expression in contrast with that of control cells (Figure 5B). Sequences of miR-378a-5p agomir, miR-378a-5p binding site on KLK4 3 '-UTR and its mutant were shown in Figure5C. Cells co-transfected with miR-378a-5p agomir and KLK4 3'-UTR showed significantly lower luciferase activity compared to cells in other three groups (Figure 5D, $\mathrm{p}<0.01$ ).

MiR-378a-5p regulates angiogenesis by targeting KLK4 and blocking Wnt/ $\beta$-catenin signaling pathway. Conditioned medium from miR-378a-5p antagomir transfected Tca-8113 cells promoted tube formation activity of HUVECs, and these effects were reversed by the medium from miR-378a-5p antagomir and KLK4 shRNA co-transfected cells (Figure 6A). Protein levels of VEGFA, Wnt1 and $\beta$-catenin were significantly higher in miR-378a-5p antagomir transfected Tca- 8113 cells than that in NC antagomir transfected Tca- 8113 cells, while expression of $p$ - $\beta$-catenin showed a reversed trend. However, these changes were all abrogated by co-transfection with KLK4 shRNA (Figure 6B). Additionally, whether miR-378-5p affects angiogenesis by the regulation of KLK4 was also investigated in the TSCCA cell line. The results showed that conditioned medium from miR-378a-5p agomir transfected cells suppressed tube formation activity of HUVECs, and these effects were reversed by the medium from miR-378a-5p agomir and KLK4 overexpression vector co-transfected cells (Figure S2C). miR-378a-5p agomir induced downregulation of VEGFA, Wnt 1 , and inactivation of the $\beta$-catenin signal were also reversed by the overexpression of KLK4 in TSCCA cells (Figure S2C). 
A

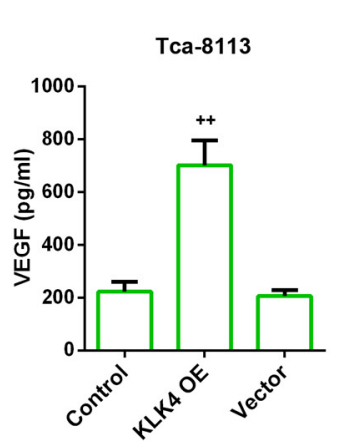

B

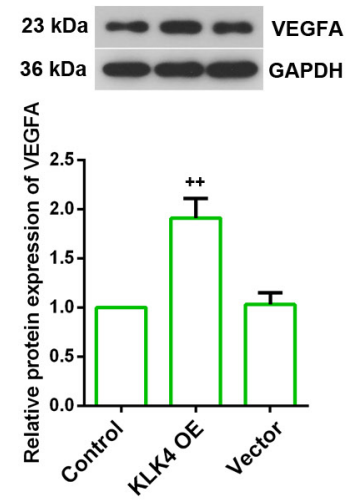

C
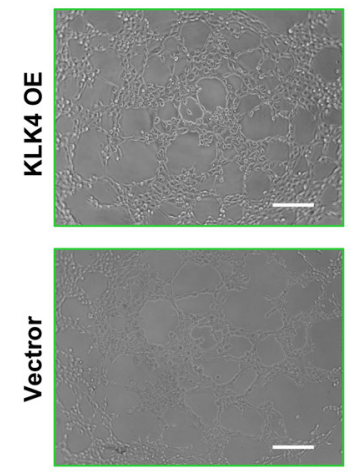

D

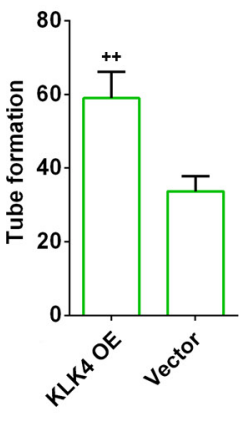

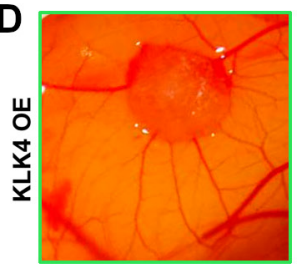

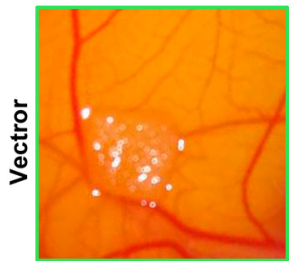

Figure 4. Effect of KLK4 on angiogenesis. A) The content of VEGF in KLK4 overexpression vector or NC vector transfected Tca-8113 cells was measured by the commercial kit. B) The protein level of VEGFA in Tca-8113 cells was determined by western blot assay. C) HUVEC were treated with the conditioned medium from KLK4 overexpression vector or NC vector transfected Tca-8113 cells, and the angiogenesis activity was detected by tube formation assay. D) The chicken chorioallantoic membrane (CAM) assay was used to assess the effect of KLK4 on angiogenesis. All data were presented as mean $\pm \mathrm{SD} .{ }^{++} \mathrm{p}<0.01$ vs. control cells.

A

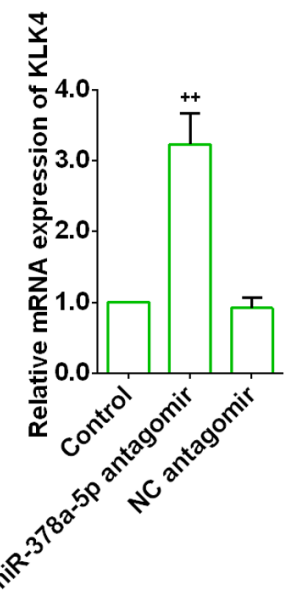

C
B
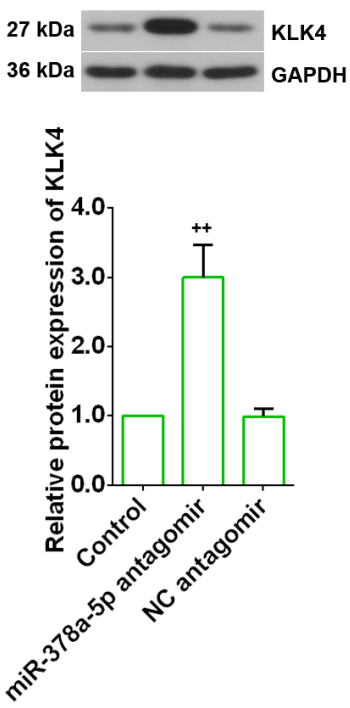
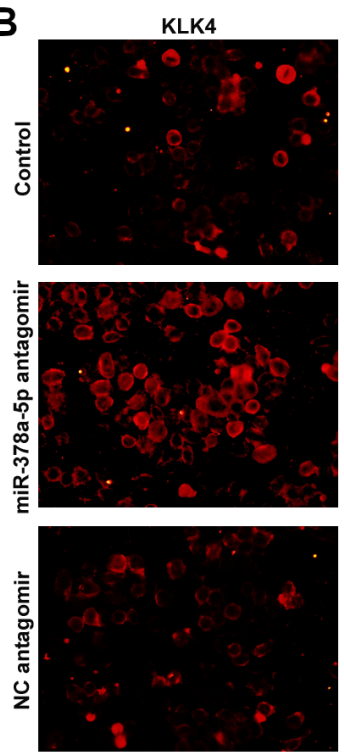

DAPI
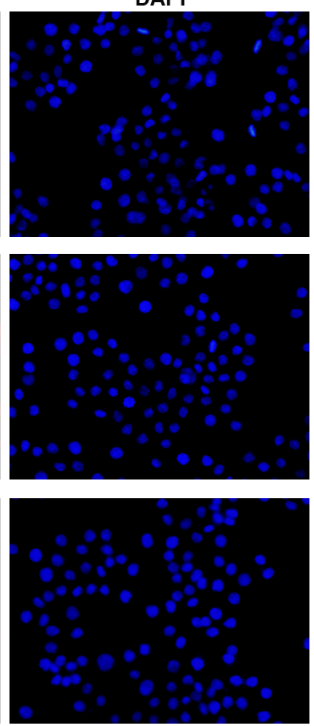
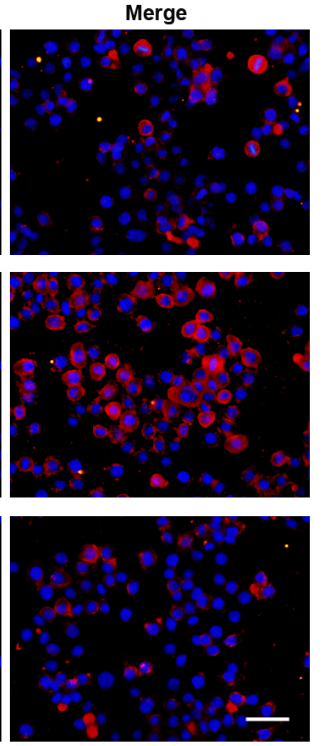

D

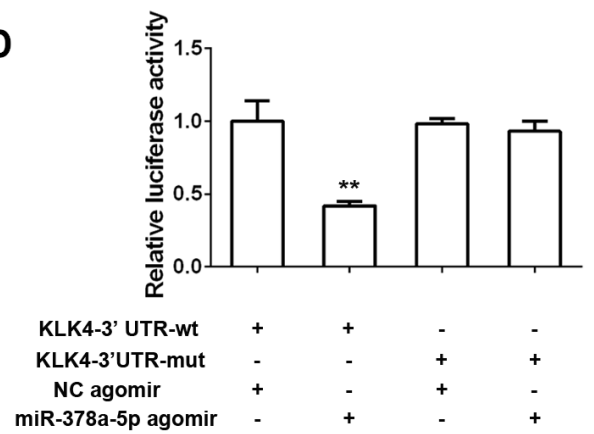

miR-378a-5p agomir 3'-UGUGUCCUGGACCUCAGUCCUC-5' KLK4 3'UTR-wT 5'-ATCCTGCGGAAGGAATTCAGGAA-3' KLK4 3'UTR-mut 5'-ATCCTGCGgAAGGATTGCTTCA-3'

Figure 5. KLK4 is a target of miR-378a-5p. A) The expression level of KLK4 in miR-378a-5p antagomir or NC antagomir transfected Tca-8113 cells was measured by quantitative real-time PCR and western blot. B) Expression of KLK4 was evaluated by immunofluorescence assay. C) The wild type and mutant sequences of KLK4 3'-UTR that targeted by miR-378a-5p. D) Regulatory effect of miR-378a-5p on KLK4 was evaluated by dual-luciferase assay. All data were presented as mean \pm SD. ${ }^{++} p<0.01$ vs. control cells.; ${ }^{++} p<0.01$ vs. KLK4-3’UTR-wt and NC agomir co-transfected cells. 


\section{Discussion}

Approximately $18 \%$ of all cancer deaths result from OSCC according to the latest cancer statistics [16]. Previous studies showed that tumor angiogenesis facilitates tumor metastasis by providing nourishment for tumor cells [17]. In this study, we attended to investigate the function of miR-378a and KLK4 on tumor angiogenesis of OSCC. Our results suggest that miR-378a-5p could inhibit whereas KLK4 could promote angiogenesis. miR-378a-5p was confirmed to negatively regulate KLK4 by targeting its 3'-UTR. Depletion of KLK4 counteracted the biological actions of miR-378a-5p knockdown in OSCC cells.

Dysregulation of miR-378 is closely associated with tumorigenesis and metastasis according to previous studies. For instance, expressions of miR-378 in glioma cells and tissues were notably reduced in contrast with that of controls. Decreased expression of miR-378 is correlated with worse clinicopathological parameters of glioma patients, while overexpression of miR-378 remarkably suppresses the epithelial-mesenchymal transition (EMT), tumor metastasis, tumor size and tumor growth rate of glioma cells [18]. The expression level of miR-378 is notably lower in pituitary adenoma (PA) tissues compared to normal tissues, besides, the proliferation and migration activities of GH3 cells are remarkably inhibited by miR-378 overexpression [19]. MiR-378 suppresses medulloblastoma cell proliferation and induces cell apoptosis by inhibiting ubiquitin-like with PHD and RING finger domains 1 expression [20]. Besides, autophagy-related protein 2 is a direct target of miR-378, indicating that miR-378 may also be implicated in autophagy [21]. MiR-378 promotes migration, invasion, and tumorigenesis of melanoma by targeting forkhead box protein $\mathrm{N} 3$ and activating Wnt/ $\beta$-catenin signaling pathway [22]. Moreover, miR-378 was reported to have a great influence on tumor angiogenesis. Proliferation and invasion ability of hepatocellular carcinoma and hepatoblastoma is inhibited by miR-378a, which also activated signaling pathway proteins

A
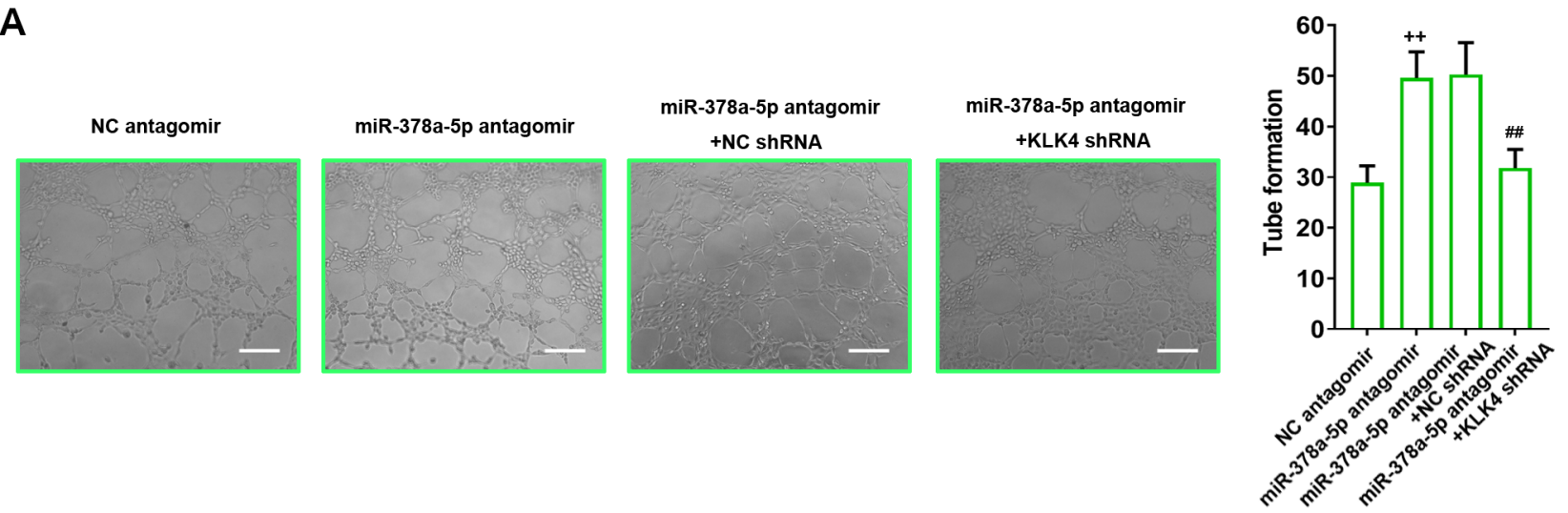

B

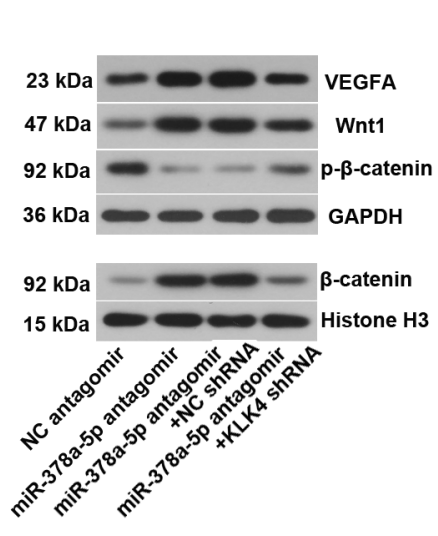

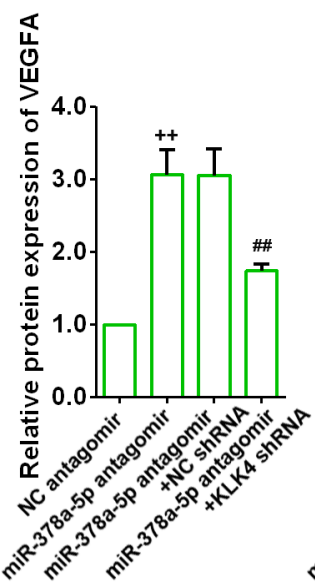

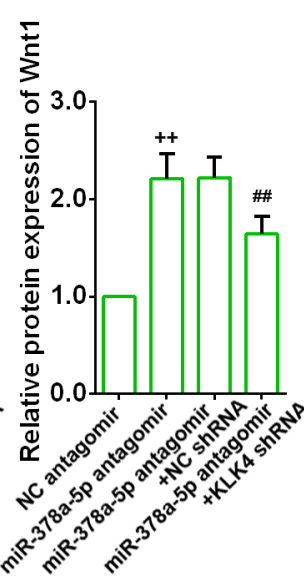

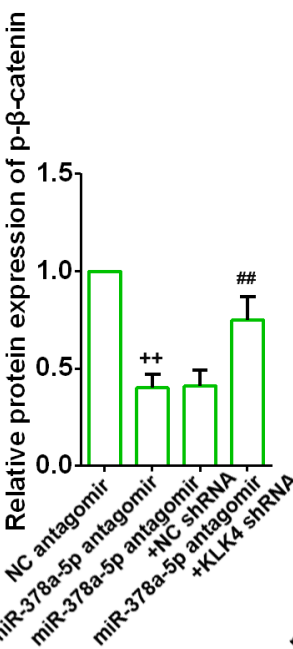

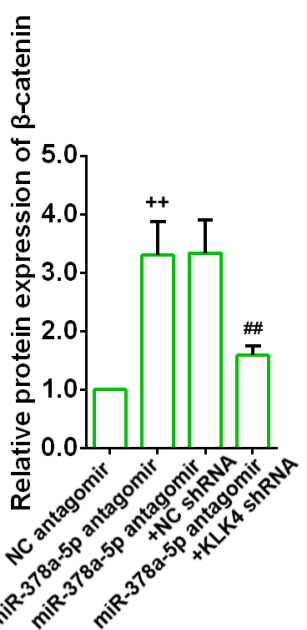

Figure 6. MiR-378a-5p regulates angiogenesis and blocking Wnt/ $\beta$-catenin signaling pathway by targeting KLK4. A) HUVECs were treated with different conditioned medium and the angiogenesis activity was determined by tube formation assay. B) The protein levels of VEGFA, Wnt 1 , p- $\beta$-catenin and intranuclear $\beta$-catenin in Tca-8113 cells were determined by western blot assay. All data were presented as mean \pm SD. ${ }^{++} p<0.01$ vs. NC antagomir transfected cells; ${ }^{* \#} \mathbf{p}<0.01$ vs. miR-378a-5p antagomir and NC shRNA co-transfected cells. 
like VEGF, one of the best-known regulators in angiogenesis and a key target in developing anti-angiogenesis therapies. Overexpression of miR-378 significantly enhances the survival of U87 cells, nude mice injected with miR-378 overexpressed U87 cells showed obviously larger tumor size [23]. Tumors generated by miR-378-transfected cells contained more blood vessels compared to those formed by vehicle transfected cells, and miR-378 exerts its function mainly by targeting suppressor of sufu, and Fus-1 [24]. By investigation in 29 matched non-small cell lung cancer patients, miR-378 is implicated in non-small cell lung cancer brain metastasis via enhancing cell migration, invasion and tumor angiogenesis [25]. Besides, miR-378 is of great importance in the clinical utilization of human cancer diagnosis and therapies. Expression level of miR-378a is closely associated with clinicopathologic characteristics including histological differentiation and TNM stage in patients with colorectal cancer (CRC). CRC patients with low miR-378a show remarkably shorter survival time than those with high miR-378a level [26-28], indicating that miR-378a may serve as a diagnostic biomarker and assist clinicians on a clinical trial for human cancers.

Influence of KLK4 on growth and metastasis of OSCC has been elucidated in our previous studies, KLK4 silencing inhibited proliferation, colony formation and induced apoptosis of OSCC cells by the regulation of Wnt/ $\beta$-catenin signaling pathway $[29,30]$. In line with our previous studies, the present study further proves the role of KLK4 in promoting angiogenesis of OSCC cells. These results indicated that KLK4 might become a promising therapeutic target for the treatment of OSCC. Besides, validated KLK4 as a target for miR-378a-5p in regulating tumor angiogenesis.

In summary, our present study showed that miR-378a-5p inhibited angiogenesis of OSCCs by targeting KLK4 and blocking Wnt/ $\beta$-catenin signaling pathway. miR-378a-5p might be a candidate therapeutic target for the treatment of OSCC.

Supplementary information is available in the online version of the paper.

\section{References}

[1] SIEGEL R, NAISHADHAM D, JEMAL A. Cancer statistics for Hispanics/Latinos, 2012. CA Cancer J Clin 2012; 62: 283298. https://doi.org/10.3322/caac.21153

[2] SIEGEL RL, MILLER KD, JEMAL A. Cancer statistics, 2016. CA Cancer J Clin 2016; 66: 7-30. https://doi.org/10.3322/ caac. 21332

[3] SANO D, MYERS JN. Metastasis of squamous cell carcinoma of the oral tongue. Cancer Metastasis Rev 2007; 26: 645-662. https://doi.org/10.1007/s10555-007-9082-y

[4] WEIS SM, CHERESH DA. Tumor angiogenesis: molecular pathways and therapeutic targets. Nat Med 2011; 17: 13591370. https://doi.org/10.1038/nm.2537
[5] WANG Z, DABROSIN C, YIN X, FUSTER MM, ARREOLA A et al. Broad targeting of angiogenesis for cancer prevention and therapy. Semin Cancer Biol 2015; 35 Suppl: S224-S243. https://doi.org/10.1016/j.semcancer.2015.01.001

[6] EICHNER LJ, PERRY MC, DUFOUR CR, BERTOS N, PARK $\mathrm{M}$ et al. miR-378(*) mediates metabolic shift in breast cancer cells via the PGC-1beta/ERRgamma transcriptional pathway. Cell Metab 2010; 12: 352-361. https://doi.org/10.1016/j. cmet.2010.09.002

[7] KRIST B, FLORCZYK U, PIETRASZEK-GREMPLEWICZ K, JOZKOWICZ A, DULAK J. The Role of miR-378a in Metabolism, Angiogenesis, and Muscle Biology. Int J Endocrinol 2015; 2015: 281756. https://doi.org/10.1155/2015/281756

[8] SCAPOLI L, PALMIERI A, LO MUZIO L, PEZZETTI F, RUBINI C et al. MicroRNA expression profiling of oral carcinoma identifies new markers of tumor progression. Int J Immunopathol Pharmacol 2010; 23: 1229-1234. https://doi. org/10.1177/039463201002300427

[9] LUNDWALL A, BAND V, BLABER M, CLEMENTS JA, COURTY Y et al. A comprehensive nomenclature for serine proteases with homology to tissue kallikreins. Biol Chem 2006; 387: 637-641. https://doi.org/10.1515/BC.2006.082

[10] PAPAGERAKIS P, PANNONE G, ZHENG LI, ATHANASSIOU-PAPAEFTHYMIOU M, YAMAKOSHI Y et al. Clinical significance of kallikrein-related peptidase- 4 in oral cancer. Anticancer Res 2015; 35: 1861-1866.

[11] LU RY, LUO DF, XIAO SH, YANG LH, ZHAO J et al. Kallikrein gene transfer induces angiogenesis and further improves regional cerebral blood flow in the early period after cerebral ischemia/reperfusion in rats. CNS Neurosci Ther 2012; 18: 395-399. https://doi.org/10.1111/j.17555949.2012.00305.x

[12] MICHAEL IP, SOTIROPOULOU G, PAMPALAKIS G, MAGKLARA A, GHOSH $\mathrm{M}$ et al. Biochemical and enzymatic characterization of human kallikrein 5 (hK5), a novel serine protease potentially involved in cancer progression. J Biol Chem 2005; 280: 14628-14635. https://doi.org/10.1074/ jbc.M408132200

[13] FUKAE M, TANABE T, SHIMIZU M. Proteolytic enzyme activity in porcine immature enamel. Tsurumi Shigaku 1977; 3: 15-17.

[14] CLEMENTS JA, WILLEMSEN NM, MYERS SA, DONG Y. The tissue kallikrein family of serine proteases: functional roles in human disease and potential as clinical biomarkers. Crit Rev Clin Lab Sci 2004; 41: 265-312. https://doi. org/10.1080/10408360490471931

[15] KRYZA T, SILVA LM, BOCK N, FUHRMAN-LUCK RA, STEPHENS CR et al. Kallikrein-related peptidase 4 induces cancer-associated fibroblast features in prostate-derived stromal cells. Mol Oncol 2017; 11: 1307-1329. https://doi. org/10.1002/1878-0261.12075

[16] SIEGEL RL, MILLER KD, JEMAL A. Cancer statistics, 2018. CA Cancer J Clin 2018; 68: 7-30. https://doi.org/10.3322/ caac. 21442

[17] SIRIWARDENA S, TSUNEMATSU T, QI G, ISHIMARU N, KUDO Y. Invasion-Related Factors as Potential Diagnostic and Therapeutic Targets in Oral Squamous Cell CarcinomaA Review. Int J Mol Sci 2018; 19. https://doi.org/10.3390/ ijms 19051462 
[18] SHI HZ, WANG D, SUN XN, SHENG L. MicroRNA-378 acts as a prognosis marker and inhibits cell migration, invasion and epithelial-mesenchymal transition in human glioma by targeting IRG1. Eur Rev Med Pharmacol Sci 2018; 22: 3837-3846. https://doi.org/10.26355/eurrev_201806_15268

[19] QIU P, XU TJ, LU XD, YANG W, ZHANG YB et al. MicroRNA-378 regulates cell proliferation and migration by repressing RNF31 in pituitary adenoma. Oncol Lett 2018; 15: 789-794. https://doi.org/10.3892/ol.2017.7431

[20] ZHANG ZY, ZHU B, ZHAO XW, ZHAN YB, BAO JJ et al. Regulation of UHRF1 by microRNA-378 modulates medulloblastoma cell proliferation and apoptosis. Oncol Rep 2017; 38: 3078-3084. https://doi.org/10.3892/or.2017.5939

[21] TAN D, ZHOU C, HAN S, HOU X, KANG S et al. MicroRNA-378 enhances migration and invasion in cervical cancer by directly targeting autophagy-related protein 12 . Mol Med Rep 2018; 17: 6319-6326. https://doi.org/10.3892/ mmr.2018.8645

[22] SUN M, MA X, TU C, WANG X, QU J et al. MicroRNA-378 regulates epithelial-mesenchymal transition and metastasis of melanoma by inhibiting FOXN3 expression through the Wnt/beta-catenin pathway. Cell Biol Int 2019; 43: 11131124. https://doi.org/10.1002/cbin.11027

[23] WU QP, XIE YZ, DENG Z, LI XM, YANG W et al. Ergosterol peroxide isolated from Ganoderma lucidum abolishes microRNA miR-378-mediated tumor cells on chemoresistance. PLoS One 2012; 7: e44579. https://doi.org/10.1371/journal. pone.0044579

[24] LEE DY, DENG Z, WANG CH, YANG BB. MicroRNA-378 promotes cell survival, tumor growth, and angiogenesis by targeting SuFu and Fus-1 expression. Proc Natl Acad Sci U S A 2007; 104: 20350-20355. https://doi.org/10.1073/ pnas.0706901104
[25] CHEN LT, XU SD, XU H, ZHANG JF, NING JF et al. MicroRNA-378 is associated with non-small cell lung cancer brain metastasis by promoting cell migration, invasion and tumor angiogenesis. Med Oncol 2012; 29: 1673-1680. https://doi.org/10.1007/s12032-011-0083-x

[26] LI H, DAI S, ZHEN T, SHI H, ZHANG F et al. Clinical and biological significance of miR-378a-3p and miR-378a-5p in colorectal cancer. Eur J Cancer 2014; 50: 1207-1221. https:// doi.org/10.1016/j.ejca.2013.12.010

[27] TANAKA H, HAZAMA S, IIDA M, TSUNEDOMI R, TAKENOUCHI $\mathrm{H}$ et al. miR-125b-1 and miR-378a are predictive biomarkers for the efficacy of vaccine treatment against colorectal cancer. Cancer Sci 2017; 108: 2229-2238. https:// doi.org/10.1111/cas.13390

[28] SHINDO Y, HAZAMA S, NAKAMURA Y, INOUE Y, KANEKIYO S et al. miR-196b, miR-378a and miR-486 are predictive biomarkers for the efficacy of vaccine treatment in colorectal cancer. Oncol Lett 2017; 14: 1355-1362. https:// doi.org/10.3892/ol.2017.6303

[29] CUI Z, CUI Y, YANG S, LUO G, WANG Y et al. KLK4 silencing inhibits the growth of oral squamous cell carcinoma through Wnt/beta-catenin signaling pathway. Cell Biol Int 2017; 41: 392-404. https://doi.org/10.1002/cbin.10736

[30] CUI Z, CUI Y, LUO G, YANG S, LING X et al. Kallikrein-related peptidase 4 contributes to the tumor metastasis of oral squamous cell carcinoma. Biosci Biotechnol Biochem 2017; 81: 1768-1777. https://doi.org/10.1080/09168451.2017.1356 216 


\section{MiR-378a-5p inhibits angiogenesis of oral squamous cell carcinoma by targeting KLK4}

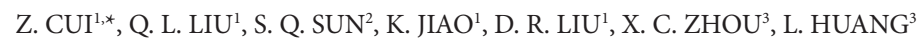

\section{Supplementary Information}

a
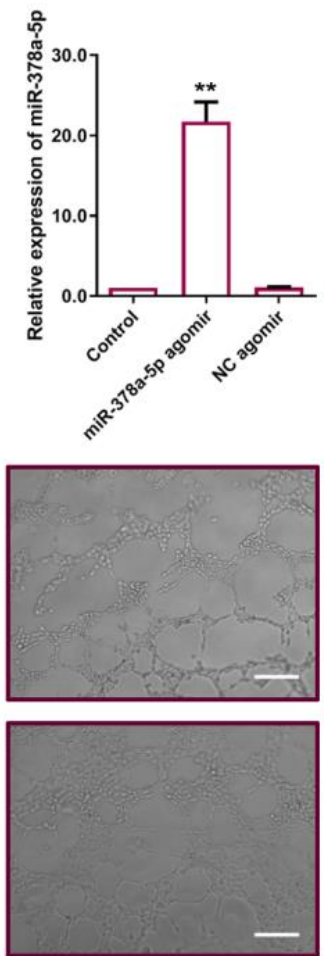
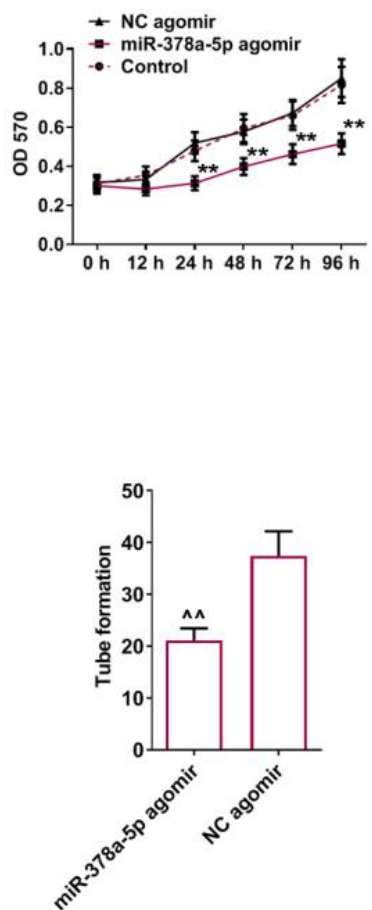
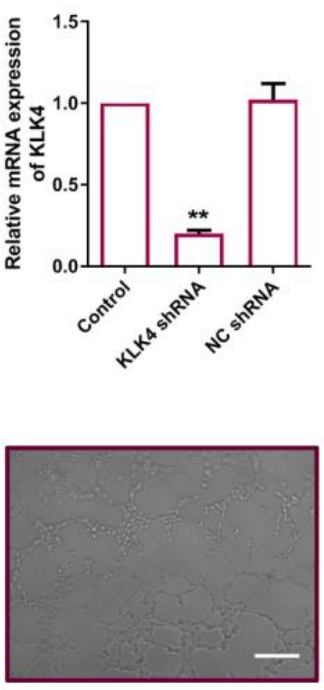
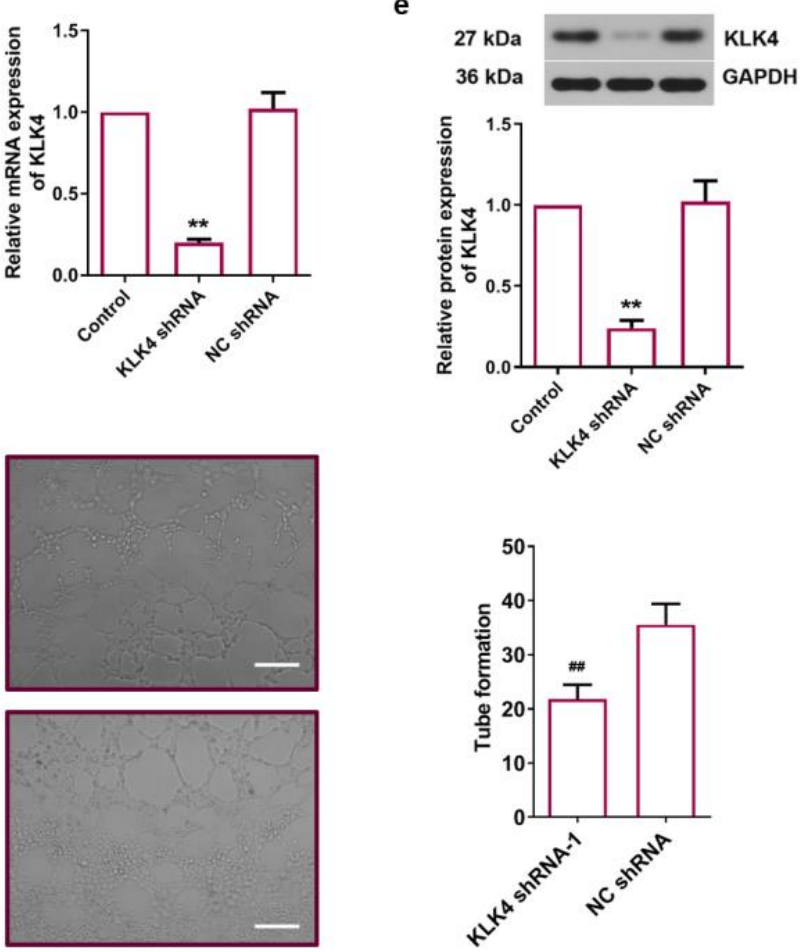

Figure S1. Effect of miR-378a-5p and KLK4 on angiogenesis. (a) Expression of miR-378a-5p in TSCCA cells was evaluated by quantitative real-time PC'R. (b) MTT assay was used to detect cell proliferation, (c) HUVECs were treated with the conditioned medium from miR-378a-5p agomir or NC agomir transfected TSCCA cells, and the angiogenesis activity was detected by tube formation assay, (d, e) The expression level of KLK4 in TSCCA cells was measured by quantitative real-time PC'R and western blot, (f) HUVECs were treated with the conditioned medium from KLK4 shRNA or NC shRNA transfected TSCCA cells, and the angiogenesis activity was detected by tube formation assay. All data were presented as mean \pm SD. ${ }^{\star \star} \mathbf{p}<0.01$ vs. control cells; $\wedge \wedge$ p $<0.01$ vs. NC agomir transfected cells; \#\#p<0.01 vs. NC shRNA transfected cells. 
a

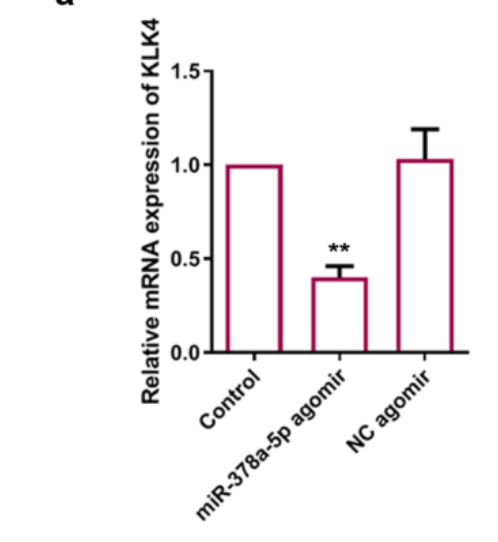

b
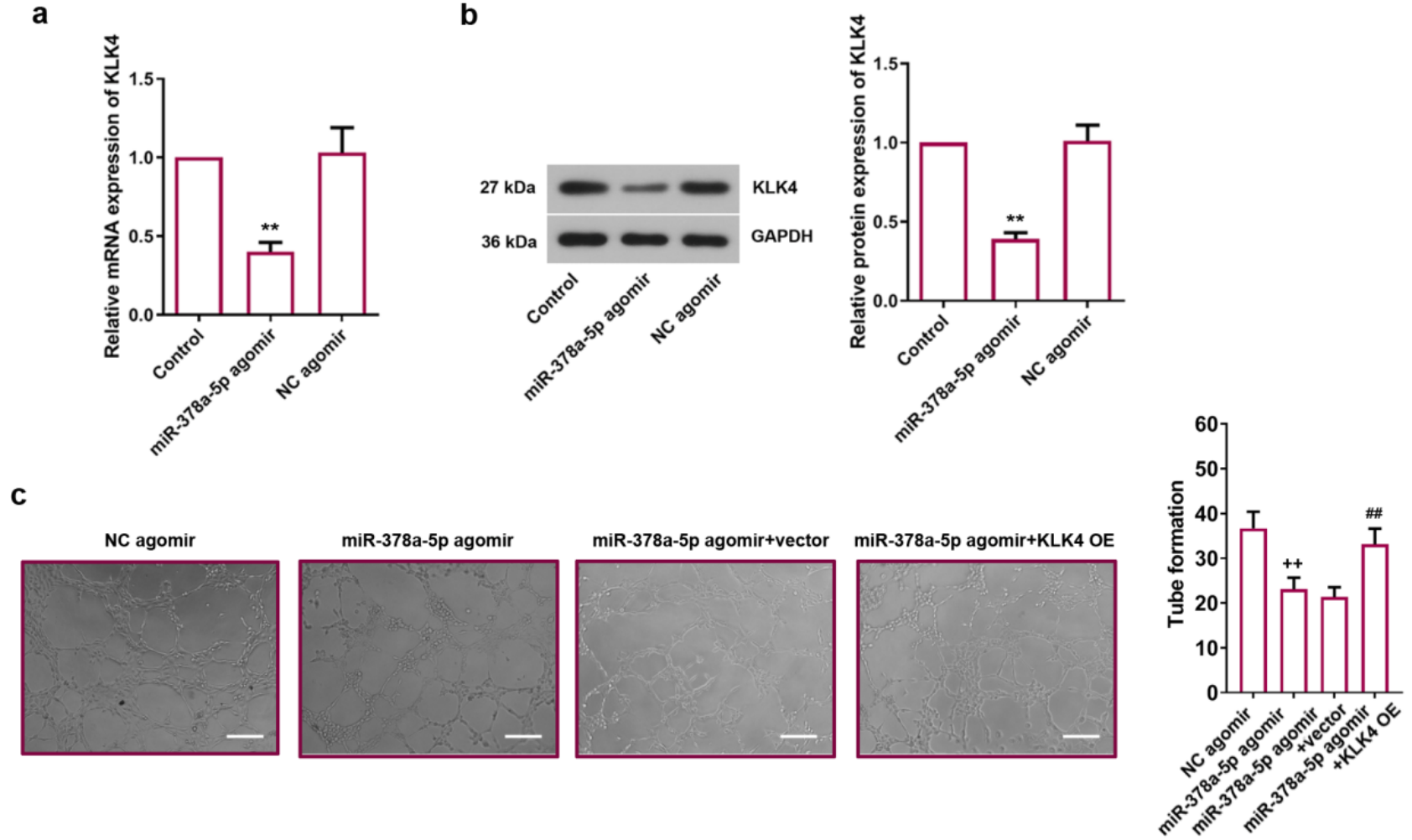

d
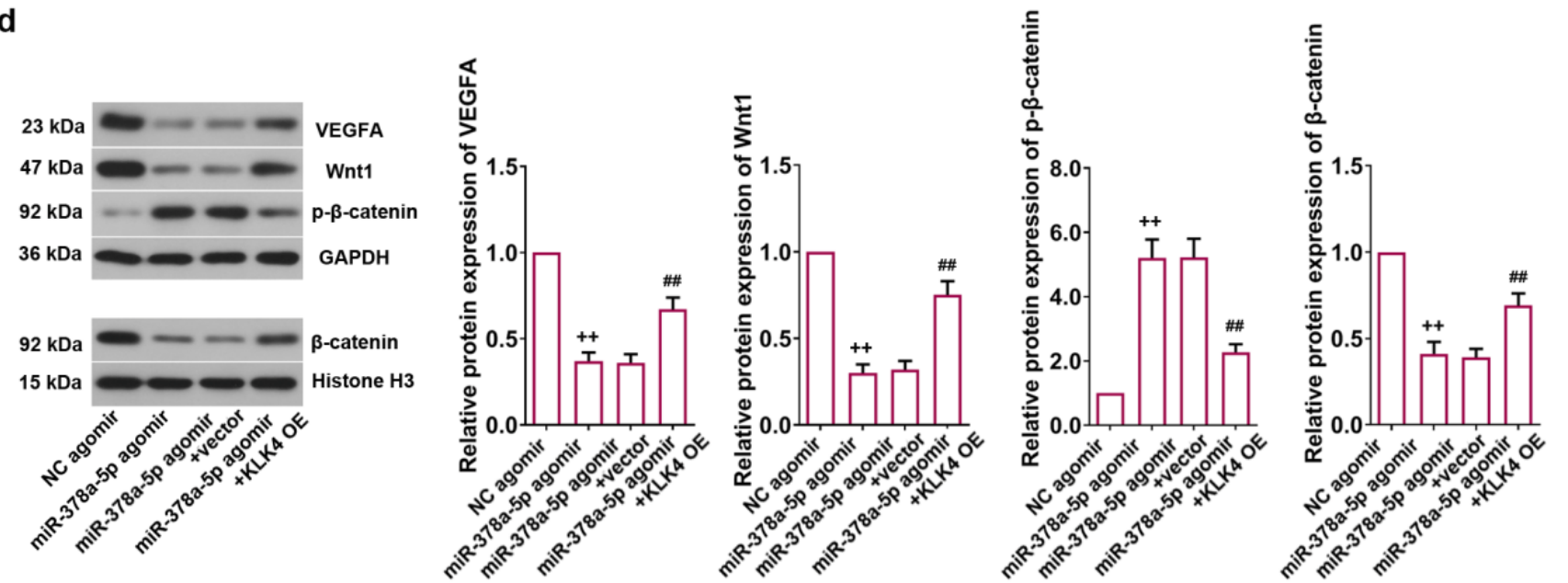

Figure S2. MiR-378a-5p regulates angiogenesis by targeting KLK4 and blocking Wut/ $\beta$-catenin signaling pathway, (a, b) The expression level of KLK4 in miR-378a-5p agomir or NC agomir transfected TSCCA cells was measured by quantitative real-time PC'R and western blot, (c) HUVECs were treated with different conditioned medium and the angiogenesis activity was determined by tube formation assay, (c) The protein levels of VEGFA, Wntl, p- $\beta$-catenin and intranuclear $\beta$-catenin in TSCCA cells were determined by western blot assay. All data were presented as mean \pm SD. ${ }^{\star *}$ p $<0.01$ vs. control cells: $++\mathbf{p}<\mathbf{0 . 0 1}$ vs. NC agomir transfected cells; \#\# $\mathbf{p}<\mathbf{0 . 0 1}$ vs. NC shRNA transfected cells. 
a

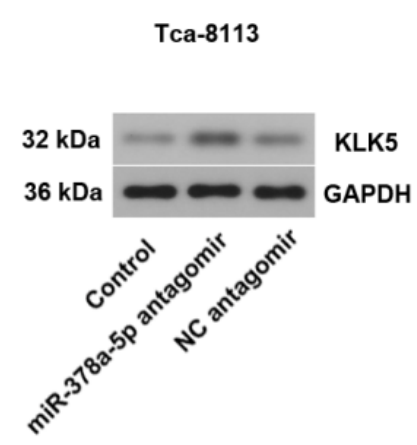

b

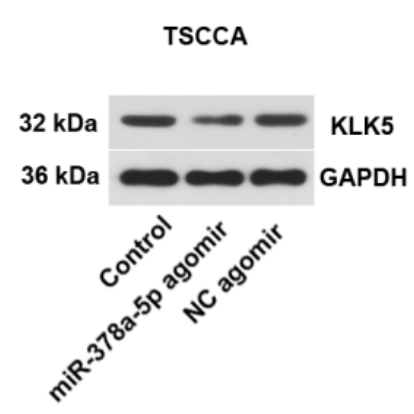

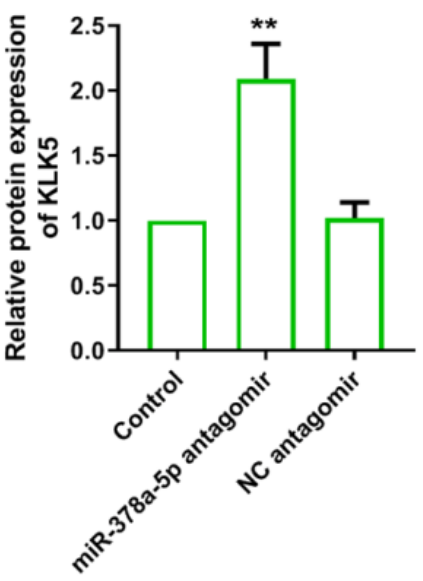

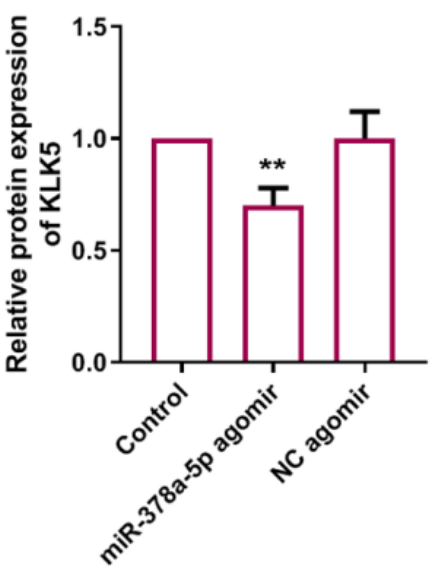

Figure S3. The expression of KLK5. The expression of KLK5 in Tca-8113 (a) and TSCCA (b) cells were determined by western blot. All data were presented as mean $\pm \mathrm{SD}$. ${ }^{* *} \mathrm{p}<0.01$ vs. control cells. 